\title{
Overshooting Effects in Nonequilibrium Ordering Dynamics
}

\section{Gilhøj, Henriette; Jeppesen, Claus; Mouritsen, Ole G.}

\section{Published in:}

Physical Review Letters

Link to article, DOI:

10.1103/PhysRevLett.75.3305

Publication date:

1995

Document Version

Publisher's PDF, also known as Version of record

Link back to DTU Orbit

Citation (APA):

Gilhøj, H., Jeppesen, C., \& Mouritsen, O. G. (1995). Overshooting Effects in Nonequilibrium Ordering Dynamics. Physical Review Letters, 75(18), 3305-3308. https://doi.org/10.1103/PhysRevLett.75.3305

\section{General rights}

Copyright and moral rights for the publications made accessible in the public portal are retained by the authors and/or other copyright owners and it is a condition of accessing publications that users recognise and abide by the legal requirements associated with these rights.

- Users may download and print one copy of any publication from the public portal for the purpose of private study or research.

- You may not further distribute the material or use it for any profit-making activity or commercial gain

- You may freely distribute the URL identifying the publication in the public portal

If you believe that this document breaches copyright please contact us providing details, and we will remove access to the work immediately and investigate your claim 


\title{
Overshooting Effects in Nonequilibrium Ordering Dynamics
}

\author{
Henriette Gilhøj, ${ }^{1}$ Claus Jeppesen, ${ }^{2}$ and Ole G. Mouritsen ${ }^{1}$ \\ ${ }^{1}$ Department of Physical Chemistry, The Technical University of Denmark, Building 206, DK-2800 Lyngby, Denmark \\ ${ }^{2}$ Materials Research Laboratory, University of California, Santa Barbara, California 93106
}

(Received 30 March 1995)

\begin{abstract}
Using Monte Carlo simulation on the simplest possible statistical mechanical model, the twodimensional, nonconserved kinetic Ising model that undergoes an order-disorder transition, we show that the local order of the ordering domains, subsequent to a temperature quench, transiently overshoots the value of the equilibrium order parameter. It is argued that overshooting is a generic effect in ordering dynamics, independent of the detailed dynamics and the conservation laws in effect.
\end{abstract}

PACS numbers: 64.60.Cn, 05.70.Ln, 64.60.My, 75.40.Mg

Nonequilibrium ordering processes are abundant in nature and include, e.g., order-disorder phenomena, phase-separation processes, spinodal decomposition, and crystallization [1]. The standard phenomenological picture of a physical system that undergoes a nonequilibrium ordering process induced by a rapid quench in temperature involves the formation of an assembly of ordered domains. At early times these domains are formed either via a nucleation event or as a consequence of a longwavelength instability (spinodal decomposition). At later times the ordered domains grow and coarsen in a way that is controlled by the diffusive motion of the complex random network of interfaces that bound the domains [2].

The theoretical description of ordering dynamics outside the early-time regime, where linearized theories can be invoked [2], is difficult because of the inherent farfrom-equilibrium nature of the process. Early classical theories suggested that a key to the problem lies in a dynamical description of diffusive domain interfaces [3], and more recently a renormalization-group treatment has provided us with a more refined picture involving dynamical scaling symmetries as well as a classification of ordering dynamics in universality classes determined by general system properties like conservation laws and symmetries [4].

The order that develops in the system in the course of the ordering process has two components, one being the actual degree of order within the domains and the other being the morphological order of the total domain pattern, specifically the average size of the domains. Hence there are two length scales in the problem, the average domain size and the correlation length of the equilibrium fluctuations of the order parameter [5,6]. Within the standard phenomenology, both types of orders proceed in a monotonous fashion from a level of low order to a level of high order. At late times the average size of the domains approaches macroscopic length scales, and it becomes the only relevant length scale of the problem. It is usually assumed that the order within the individual domains very early approaches that of the equilibrium state, i.e., the local order of the domain is described by the value of the equilibrium global order parameter.

$0031-9007 / 95 / 75(18) / 3305(4) \$ 06.00$
In the present Letter we shall provide definite numerical evidence from an actual and detailed model calculation which demonstrates that this latter assumption is generally not correct. Instead, the local order of the growing domains at early times overshoots the equilibrium order parameter and only later decays towards the equilibrium order. Hence, the local domain order parameter varies in a nonmonotonous fashion. Qualitatively, this finding is robust to variations in the microscopic dynamics as well system parameters and thermodynamic conditions. The degree of overshooting is found to increase with temperature, which strongly suggests that the effect is of entropic origin. By combining our results with previous findings for ordering dynamics in other models, we put forward the hypothesis that overshooting is a generic property of nonequilibrium ordering processes and is governed by a competition between nonequilibrium internal energy and entropy: The energy, being a local property, is easily minimized at early times by fast dissipation of heat into the heat bath. This leads to a high degree of local order within the domains. Still, the total nonequilibrium entropy decreases monotonically. The disorder becomes localized in the domain-boundary network. Only at much later times is the high local domain order and the corresponding low domain entropy balanced by an increased disorder which dissolves into the domains as the interfacial density diminishes. This latter stage is a slow process since it is controlled by the maximization of the total entropy that is a global property and hence only manifests itself over longer times.

Our results are obtained using the simplest possible statistical mechanical lattice model with a nontrivial orderdisorder transition, the two-dimensional, nearest-neighbor ferromagnetic Ising model defined by the Hamiltonian

$$
\mathcal{H}=-J \sum_{\langle i, j\rangle} \sigma_{i} \sigma_{j},
$$

with $J>0$ and $\sigma_{i}= \pm 1$. The model is arrayed on a triangular lattice with $N=L \times L$ sites and with periodic boundary conditions. Finite-size effects have been assessed by studying a series of different lattice sizes, $N=200^{2}, 500^{2}$, and $1000^{2}$.

(C) 1995 The American Physical Society 
The microscopic dynamics of the model is taken to be single-site Glauber dynamics, corresponding to single spin-flip attempts (the kinetic Ising model). By this dynamics the global order parameter, $\Psi=\left\langle N^{-1} \sum_{i} \sigma_{i}\right\rangle$ (the magnetization), is a nonconserved field. The dynamic evolution of the system is simulated by standard Monte Carlo simulation with an acceptance criterion that involves the Metropolis function, $\min \left(1, \exp \left(-\Delta \mathcal{H} / k_{B} T\right)\right)$, where $\Delta \mathcal{H}$ is the total internal energy associated with the update. We have checked that our results do not qualitatively depend on this choice by repeating some of the simulations using the symmetric Glauber function, $\frac{1}{2}\left[1-\tanh \left(\Delta \mathcal{H} / 2 k_{B} T\right)\right]$.

The quenches are performed by initiating the system in the completely random $(T=\infty)$ state and then changing the temperature to a value below the critical point. The time is measured in units of Monte Carlo steps (MCS) per lattice site. The temporal evolution of order in the system is monitored in several different ways. Firstly, the order reflecting the domain organization of the system is monitored via calculation of the excess energy, $\Delta E(t)=\mathcal{H}(t)-\langle\mathcal{H}\rangle_{T}$, which for localized domain boundaries is a measure of the total length of the domainboundary network. Therefore, by scaling [7], the inverse excess energy is a measure of the average linear domain size, $R_{E}(t)$,

$$
R_{E}(t)=\langle\mathcal{H}\rangle_{T} /\left[\overline{\mathcal{H}(t)}-\langle\mathcal{H}\rangle_{T}\right] .
$$

$\langle\mathcal{H}\rangle_{T}$ is the internal energy in thermodynamic equilibrium at temperature $T$. In order to provide ensemble averages, indicated by the overbar in Eq. (2), the timedependent quantities have been averaged over a large number (typically 10-50 depending on system size) of independent quenches. Secondly, in order to provide a measure of the actual local spin order within the domains, we have calculated the average domain order parameter (or domain magnetization), $\Psi_{\text {loc }}(t)$. For that purpose we define any set of lattice points that are spanned by a nearestneighbor-connected cluster of spins in the same spin state as an ordered domain provided that the number of sites exceeds a certain minimum number (here taken to be four sites, a choice that does not qualitatively affect the results). By this definition, a domain may internally contain clusters of up to three connected sites with spins of the opposite spin value (excitations). In quantitative terms the average domain order parameter can be expressed as

$$
\Psi_{\text {loc }}(t)=\overline{\left(\sum_{k}^{M} N_{k}\right)^{-1} \sum_{k=1}^{M}\left|\sum_{i_{k}=1}^{N_{k}} \sigma_{i_{k}}\right|},
$$

where $M$ is the number of separate, ordered domains that have been found for a given configuration, and the order parameter of each of these domains, $k$, is determined by summing over all, $N_{k}$, lattice sites, $i_{k}$, within that domain. Hence, the contributions to the average in Eq. (3) from the different domains are weighted according to their area. Only domains exceeding a certain minimum size are included in Eq. (3). We have somewhat arbitrarily chosen this minimum size to be 100 sites. This choice does not qualitatively affect the results obtained. By symmetry the global order parameter, i.e., the total magnetization, is zero at all times. We shall define the thermodynamic limit of the equilibrium order parameter, $\Psi(T)$, as that of a symmetry-broken one-domain state. With that definition, $\Psi(T)$ becomes very close to the long-time limit of the local domain order parameter defined above in Eq. (3), i.e., $\Psi_{\text {loc }}(t \rightarrow \infty) \simeq \Psi(T)[8]$.

In Fig. 1 the results are shown for the time dependence of the average linear domain size $R_{E}(t)$ as calculated from Eq. (2) for three different quench temperatures. It is observed for all three temperatures that the domain growth over several decades follows an algebraic growth law, $R_{E}(t) \sim t^{n}$, with exponent values in the range $n \simeq 0.44-$ 0.49. $n$ approaches $1 / 2$ as the temperature is lowered. This is the expected and well-known Lifshitz-Allen-Cahn growth exponent for nonconserved ordering dynamics [2]. Hence, the global order in terms of the typical domain size and degree of organization increases monotonically and algebraically with time.

This is not the case for the average domain order parameter, $\Psi_{\text {loc }}(t)$, as demonstrated in Figs. 2 and 3. This measure of local spin order within the domains varies nonmonotonically and displays a distinct maximum in the early-time regime. This maximum is above the equilibrium value of the order parameter. In the time regime beyond this maximum, the order of domains decreases slowly in time towards its equilibrium value, $\Psi(T)$. We shall in the following refer to this phenomenon as an "overshooting effect." The finding of overshooting in the local domain order constitutes the main result of the present paper.

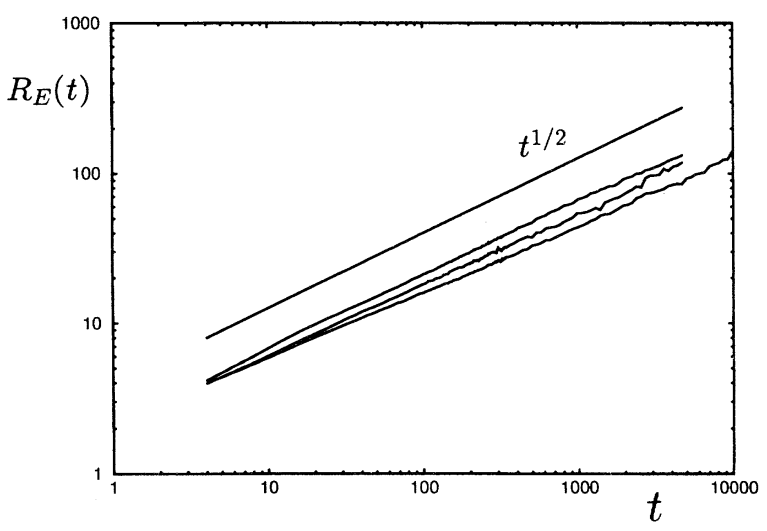

FIG. 1. Double-logarithmic plot of the average linear length scale, $R_{E}(t)$, calculated from the excess energy according to Eq. (2), as a function of time $t$ (in units of MCS). The results are obtained from quenches of the nonconserved Ising model from infinite temperature to a temperature $T / T_{\mathrm{c}}$ below the critical temperature $T_{\mathrm{c}}$ using a lattice with $N=200^{2}$ sites. Results are shown for three different temperatures, $T / T_{\mathrm{c}}=$ $0.8,0.7$, and 0.5 (curves from bottom up). The thin solid line represents the power law $t^{1 / 2}$. 


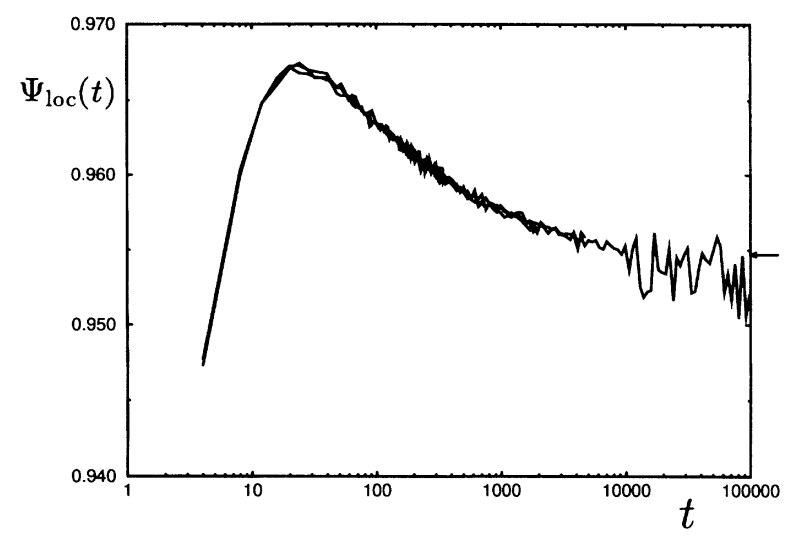

FIG. 2. Semilogarithmic plot of the average domain order parameter, $\Psi_{\text {loc }}(t)$ in Eq. (3), as a function of time $t$ (in units of MCS). The data are obtained from quenches of the nonconserved Ising model from infinite temperature to a temperature, $T / T_{\mathrm{c}}=0.8$, below the critical temperature $T_{\mathrm{c}}$. The three different curves correspond to three different lattice sizes, $N=200^{2}, 500^{2}$, and $1000^{2}$. The arrow to the right indicates the corresponding equilibrium value, $\Psi_{\mathrm{loc}}(t \rightarrow \infty)$. Beyond $t \sim 10^{4}$ only data for the smaller system are displayed. These late-time data display more scatter since they refer to averages over fewer runs than the early-time data.

It is clear from the data presented for different system sizes in Fig. 2 that the overshooting effect is not a finitesize effect in the sense that it is sensitive to the size of the lattice used. Furthermore, it is not a dynamical finitesize effect caused by the time-dependent finite size of the evolving domains. Since the domains that enter the definition of the local order parameter in Eq. (3) are of finite size, their order, if in equilibrium, is different from that of an infinite system, as predicted by finite-size scaling theory [9]. However, since these domains are subject to boundary conditions that impose either disorder or the op-

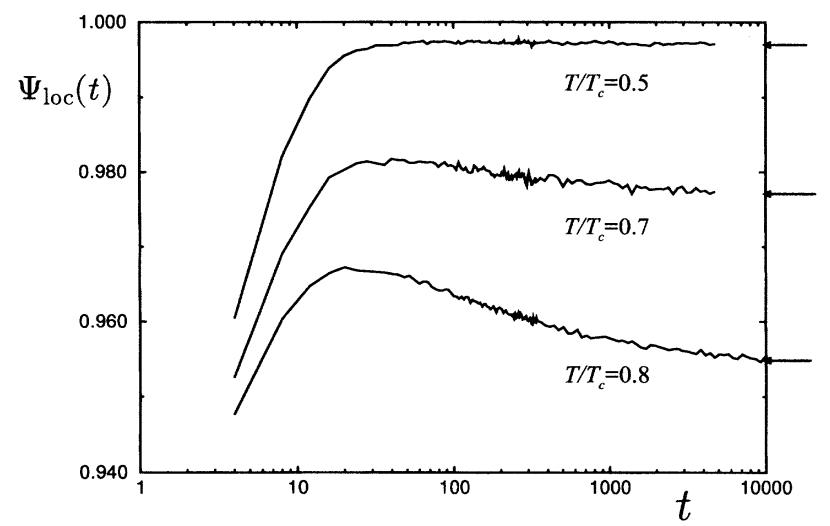

FIG. 3. Semilogarithmic plot of the average domain order parameter, $\Psi_{\text {loc }}(t)$ in Eq. (3), as a function of time $t$ (in units of MCS). Results are shown as obtained from quenches of the nonconserved Ising model from infinite temperature to three different temperatures, $T / T_{\mathrm{c}}=0.5,0.7$, and 0.8 , below the critical temperature $T_{\mathrm{c}}$ using a lattice with $N=200^{2}$ sites. The arrows to the right indicate the corresponding equilibrium values, $\Psi_{\text {loc }}(t \rightarrow \infty)$, for the three different temperatures. posite magnetization, any finite-size rounding would tend to lower rather than increase the finite-size equilibrium order of the domain. Hence, the overshooting effects seen in Figs. 2 and 3 are possibly somewhat suppressed by these time-dependent finite-size effects.

As we have pointed out above, the overshooting effect is robust to the choice of simulation algorithm as well as details regarding the actual definition of the local measure of order. Figure 3 demonstrates that the degree of overshooting increases with temperature. This is an important result which supports our assertion that the effect is likely to be of entropic origin. The slight but systematic temperature dependence of the growth exponent derived from Fig. 1 is likely to be caused by the overshooting effect. Since the order within the domains is larger than the equilibrium order, it follows that the domain energy is lower than the equilibrium energy. Hence the assumption underlying the use of $R_{E}(t)$ in Eq. (2) as a length-scale measure is not fully correct, i.e., the excess energy is not entirely located in the domain boundaries. However, as the temperature is lowered the overshooting effect diminishes and the growth exponent approaches the Lifshitz-Allen-Cahn value; cf. Fig. 1.

The picture we are left with after these results is one according to which the system at early times forms ordered domains with a length scale that increases monotonically with time. The order within the domains, however, becomes at an early time larger than the value of the thermodynamic equilibrium order parameter at the temperature in question. Disorder in terms of thermal defects like spin excitations is transiently expelled from the ordered domains and accumulated at the domain interfaces. Only very slowly does this disorder dissolve into the bulk domains as the domain-boundary network is annealed.

We have further investigated the overshooting effect by introducing an annealed site dilution in the model. It is found, in the case of low global and fixed vacancy concentrations $(\sim 1 \%)$ and for quenches into the dilute Ising-ordered phase, that the overshooting in the domain order becomes even more pronounced (data not shown) and that, concomitantly with this effect, a depletion effect occurs in the local vacancy concentration within the domains. The local domain vacancy concentration is found to go through a minimum corresponding to the maximum in the local spin order. The vacancies are transiently expelled from the ordered domains and segregate at the domain interfaces. Only at later times do the vacancies dissolve again into the ordered domains as the entropy of mixing manifests itself.

We have reasons to believe that overshooting is a generic effect in nonequilibrium ordering dynamics, and we wish to hypothesize that it is caused by a competition between nonequilibrium internal energy and nonequilibrium entropy. The system can easily at early times reduce its internal energy by local rearrangements which lead to a high degree of local order. The entropic contribution to the free energy only manifests itself at later times 
since it is a global system property which requires sampling over a large part of phase space. The pronounced increase of the overshooting with increasing temperature supports the entropic nature of the effect. Similar overshooting effects have recently been seen in studies of other two-dimensional models, including the conserved two- and three-state Potts model [10] as well as nonconserved high$q$ Potts models with site dilution [11]. Hence it appears that overshooting is a general effect in ordering dynamics, for both conserved and nonconserved systems. Within the field of critical relaxation, theory as well as simulations have indicated a kind of overshooting effect in the global order parameter related to the decay of the correlations built into the initial disordered state [12]. Since these types of correlations are induced by external fields not present in our model, it is unclear as to how the two types of overshooting are related. In any case it would be interesting to extend the present type of calculations to include quenches to the critical point in order to maximize the overshooting.

We are aware of only a few experimental results on ordering dynamics which provide evidence of overshooting effects. Firstly, using time-resolved x-ray synchrotron scattering, Park et al. [13] were able to separate the early-time short- and long-range order components of the nonconserved ordering process in $\mathrm{Fe}_{3} \mathrm{Al}$ and found that, whereas the long-range order increased monotonically with time, the short-range order (described by the correlation length of the order-parameter fluctuations) went through a maximum. This observation was taken as support for the time-dependent Ginzburg-Landau theory developed by Mazenko, Valls, and Zanetti [5] for the structure factor in the case of nonconserved ordering dynamics. Morin, Elder, and Grant [6] have argued, using a phenomenological Langevin approach, that the experimental results by Park et al. may be explained as due to a subtle coupling between short- and long-range order without the involvement of domain boundaries. Secondly, fluorescence measurements [14] as well as model simulation studies [15] of the phase-separation dynamics in binary phospholipid bilayer mixtures have also provided strong indirect evidence of transient ordering and overshooting effects in the acyl-chain order of one of the demixing species. Finally, some numerical work related to the spinodal decomposition process in a bcc TiV alloy indicated the onset of transiently ordered states at early times which were decaying again as the separation process proceeded [16,17]. The reason for the scarcity of experimental data to assess the presence of overshooting effects may partly be due to limits in time resolution and partly to a preoccupation with the late-time behavior.

Because of the general nature of the overshooting effects discussed in the present Letter, one would expect such effects to be present in many systems undergoing spontaneous ordering processes subsequent to abrupt changes in thermodynamic conditions. An interesting case to consider is that of structure formation and fold- ing of polymers and macromolecules, such as proteins, where overshooting could imply early-time formation of secondary structural elements with a high degree of local order facilitated by saturation of local interaction bonds, which later again becomes somewhat disordered due to tertiary-stucture formation and global entropy maximization in the near-equilibrium state.

This work was supported by the Danish Natural Science Research Council under Grant No. 11-0065-1 and partially supported by the MRL Program of the National Science Research Foundation under Award No. DMR9123048. Useful comments from Hans C. Fogedby and Jacob Juhl Christensen are gratefully acknowledged.

[1] Dynamics of Ordering Processes in Condensed Matter, edited by S. Komura and H. Furukawa (Plenum, New York, 1988).

[2] J.D. Gunton, M. San Miguel, and P.S. Sahni, in Phase Transitions and Critical Phenomena, edited by C. Domb and J. L. Lebowitz (Academic Press, New York, 1983), Vol. 8, p. 267.

[3] I. M. Lifshitz, Sov. Phys. JETP 15, 939 (1962) [Zh. Eksp. Teor. Fiz. 42, 1354 (1962)]; S. M. Allen and J. W. Cahn, Acta Metall. 27, 1085 (1979); I. M. Lifshitz and V.V. Slyozov, J. Phys. Chem. Solids 19, 35 (1961).

[4] A. J. Bray, Adv. Phys. 43, 357 (1994).

[5] G. F. Mazenko, O. Valls, and M. Zanetti, Phys. Rev. B 38, 520 (1988); G. F. Mazenko, Phys. Rev. Lett. 63, 1605 (1989); G. F. Mazenko, Phys. Rev. B 42, 4487 (1990).

[6] B. Morin, K. R. Elder, and M. Grant, Phys. Rev. B 47, 2487 (1993).

[7] K. Binder and D. Stauffer, Phys. Rev. Lett. 33, 1006 (1974).

[8] Because of the choice of a lower cutoff in the definition of $\Psi_{\text {loc }}(t)$, which is necessary in order to separate domains from spin excitations, $\Psi_{\text {loc }}(t \rightarrow \infty)$ is not identical to the thermodynamic order parameter $\Psi(T)$. However, the difference between the two is very small for the temperatures studied here. Close to the critical point, where large excitations involving many spins are formed, the difference becomes large.

[9] K. Binder, in Finite Size Scaling and Numerical Simulation, edited by V. Privman (World Scientific, Singapore, 1990), p. 173.

[10] C. Jeppesen and O. G. Mouritsen, Phys. Rev. B 47, 14724 (1993).

[11] H. Gilhøj, C. Jeppesen, and O. G. Mouritsen, Phys. Rev. E 52, 1465 (1995).

[12] H. K. Janssen, B. Schaub, and B. Schmittmann, Z. Phys. B 73, 539 (1989); D. A. Huse, Phys. Rev. B 40, 304 (1989).

[13] B. Park, G. B. Stephenson, S. M. Allen, and K. F. Ludwig, Phys. Rev. Lett. 68, 1742 (1992).

[14] A. Klinger, M. Braiman, K. Jørgensen, and R. L. Biltonen, Biophys. J. 66, A173 (1994).

[15] O. G. Mouritsen and K. Jørgensen, Chem. Phys. Lipids 73, 3 (1994).

[16] L.-Q. Chen and A.G. Khachaturyan, Phys. Rev. B 44, 4681 (1991).

[17] L. Reinhard and P. E. A. Turchi, Phys. Rev. Lett. 72, 120 (1994). 